\title{
Nonlinear Pre-Distortion Based on Indirect Learning Architecture and Cross-Correlation-Enabled Behavioral Modeling for 120-Gbps Multimode Optical Interconnects
}

\author{
Chenyu Liang, Wenjia Zhang*, Ling Ge, Jiangbing Du and Zuyuan He \\ State Key Laboratory of Advanced Optical Communication Systems and Networks, \\ Shanghai Jiao Tong University, Shanghai, China, 200240 \\ *e-mail: wenjia.zhang@sjtu.edu.cn
}

\begin{abstract}
In this paper, we present a novel nonlinear pre-distortion scheme enabled by indirect learning architecture and cross-correlation based behavioral modeling. 120-Gbps PAM-4 error free transmission is demonstrated using 30-GHz class VCSEL. () 2020 The Author(s)
\end{abstract}

\section{Introduction}

As the society is stepping into the era of information, there emerges evolutionary development of new technologies, such as 5G, AR, VR and HD video. The urgent needs for high speed short-reach data transmission [1] have been the fuel for engine of the development of optical interconnects. As the required data rate for single lane approaching 100 Gbps and $200 \mathrm{Gbps}$ to achieve aggregate $400 \mathrm{G}$ even towards $800 \mathrm{G} / 1 \mathrm{~T}$ transmission capacity, the impact of nonlinearities of optical and electrical devices would become a significant factor for the high speed signals. Thus, it is essential to characterize the nonlinearity of devices and mitigate the impacts of nonlinearities on the signal quality for high speed transmissions.

There are many studies investigating methods to mitigate the nonlinearities of optical and electrical devices, such as receiver-side equalization using Volterra nonlinear equalizer (VNLE) and artificial neural network (ANN) [2-4] and transmitter-side pre-distortion [5]. As indicated in [6,7], the nonlinear pre-distortion scheme would be more attractive than the receiver-side nonlinear equalization for the following reasons: (1) The SNR at transmitter side is higher than that at receiver side, thus the coefficients estimation would be more accurate at the transmitter side; (2) nonlinear pre-distortion is capable of dealing with the nonlinear distortions before the signal is further distorted by other distortions in the transmission channel. In order to calculate the nonlinear pre-distortion coefficients, the indirect learning architecture (ILA) [8] is proposed for its convenience. In the ILA, in order to process the data offline to avoid real-time processing, the nonlinear characteristic of the devices emulated with Volterra series is proposed where the kernels are obtained using least square (LS) fitting method [5, 9]. This fitting method would lead to numerical instability when identifying and extracting the Volterra kernels that describe the nonlinearity of devices [10]. It also requires high computational complexity since in the process of optimizing memory length for each order of kernels to fit the response of the system, each memory length will require a new training process. Kernel estimation based on behavioral modeling [11] using cross-correlation method [12] can directly obtain the kernel distribution for the nonlinear system without need of iterative training, therefore, it can eliminate the shortcomings of the LS fitting method when determine the kernels that emulate the nonlinear system. There also exists pre-distortion scheme using rate equation for vertical-cavity surface-emitting laser (VCSEL) [13] as well as micro-ring modulator (MRM) [14]. However, the rate-equation based pre-distortion scheme cannot compensate nonlinear distortion for the whole link with the receiver included. Behavioral modeling can characterize the nonlinearity of devices as well as the transmission link, therefore pre-distortion based on behavioral modeling can compensate the nonlinear distortion induced by the whole transmission link. There are no studies investigating the behavioral modeling based pre-distortion for the VCSEL based optical interconnects when data rate exceeds 100 $\mathrm{Gb} / \mathrm{s}$ when device bandwidth is around $30 \mathrm{GHz}$ which is an available bandwidth in the current state [15].

In this paper, we propose a nonlinear pre-distortion scheme based on ILA and cross-correlation-enabled behavioral modeling for 120 -Gbps multimode optical interconnects. The pre-distortion coefficients are obtained using ILA after the kernels of the Volterra series that emulate the nonlinearity of VCSEL are obtained by crosscorrelation based behavioral modeling [11, 12]. By applying the proposed pre-distortion scheme, 120-Gbps PAM-4 error free transmission is successfully demonstrated using 30-GHz class VCSEL.

\section{Principle of Pre-Distortion Assisted by ILA and Cross-Correlation-Enabled Behavioral Modeling}

The kernel estimation of the nonlinear system is based on cross-correlation method when the input of the system is white Gaussian noise [12]. The kernels obtained by cross-correlation method are called Wiener kernels. In this work the nonlinear system is expressed using Volterra kernels, in which only first three orders are considered. The relationship and transform between Volterra and Wiener kernels can be found in [16]. In the pre-distortion system, 
Table 1. Simulation Parameters in VPI

\begin{tabular}{c|c||c|c||c|c}
\hline Parameter & Value & Parameter & Value & Parameter & Value \\
\hline Noise Signal Bandwidth & $240 \mathrm{GHz}$ & VCSEL Photon Lifetime & $4 \mathrm{ps}$ & VCSEL Bias & $8 \mathrm{~mA}$ \\
\hline Noise Signal Standard Deviation & $6 \mathrm{~mA}$ & VCSEL Parasitic Bandwidth & $30 \mathrm{GHz}$ & PD Responsivity & $1 \mathrm{~A} / \mathrm{W}$ \\
\hline Driving Signal Swing & $6 \mathrm{~mA}$ & VCSEL Gain Parameter & $4000 / \mathrm{cm}$ & PD Bandwidth & $30 \mathrm{GHz}$ \\
\hline VCSEL Oxide Aperture & $5 \mu \mathrm{m}$ & Wavelength & $850 \mathrm{~nm}$ & & \\
\hline
\end{tabular}

Volterra kernels with first three orders are also utilized. After characterizing and emulating the nonlinear system, the kernels of pre-distortion Volterra series are calculated using ILA. The steps of ILA are expressed as follows [8]. At the first iteration, the input signal passes through the pre-distorter with initial coefficients distribution (the first value of the $1^{\text {st }}$ order kernel is set to 1 . The rest of the coefficients of $1^{\text {st }}$ order as well as coefficients of the whole $2^{\text {nd }}$ and $3^{\text {rd }}$ order kernels of the pre-distortion Volterra series are set to 0$)$. The pre-distorted signal $\boldsymbol{d}(\boldsymbol{n})$ then passes through the emulated nonlinear system $\boldsymbol{H}$ where the kernels are determined using behavioral modeling. The output $\boldsymbol{y}(\boldsymbol{n})$ is treated as the input of the pre-distorter and the corresponding output $\boldsymbol{o}(\boldsymbol{n})$ is subtracted by $\boldsymbol{d}(\boldsymbol{n})$. The error $\boldsymbol{e}(\boldsymbol{n})$ is used to train the coefficients of the pre-distorter using Least Mean Square (LMS) algorithm.
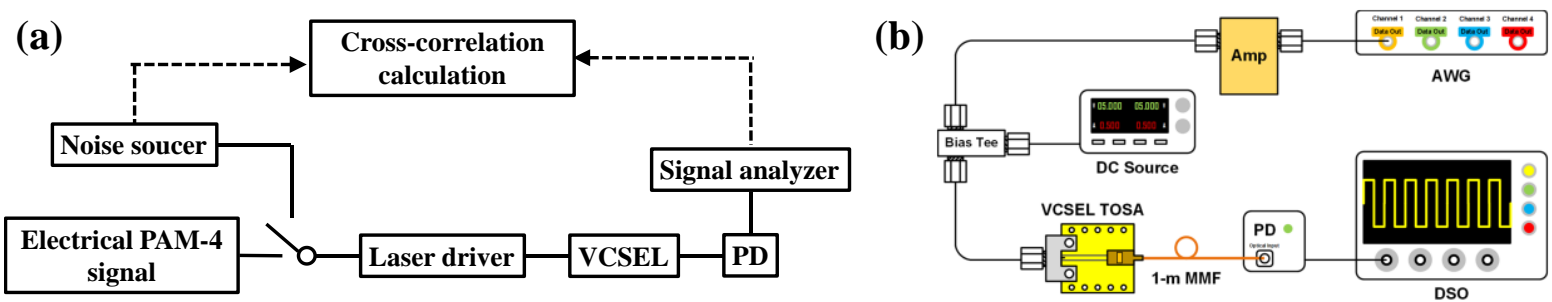

Fig. 1. (a) Simulation and (b) experimental setup for cross-correlation based behavioral modeling verification. AWG: arbitrary waveform generator. TOSA: transmitter optical sub-assembly. Amp: amplifier. PD: Photodetector. DSO: digital storage oscilloscope.

\section{Results and Discussions}

We first verify the validity of the cross-correlation-based behavioral modeling in both simulation and experiment. The simulation is performed in VPI. The simulation setup is shown in Fig. 1(a) and the parameters used in the simulation are listed in Table. 1. First the Gaussian white noise source is chosen as the input of the VCSEL module in VPI and the response after PD is sent to computer to calculate the cross-correlation with the input noise. The memory length of kernels of the Volterra series that emulate the nonlinear performance of VCSEL and PD are adjusted to ensure the best large signal response fitting. The estimated Volterra kernels are depicted in Fig. 2(a)-(c). From Fig. 3(d), it can be seen that the calculated eye diagram using the estimated Volterra kernels for 50-Gbps PAM-4 signal fits very well with the signal obtained directly from the PD output in simulation.

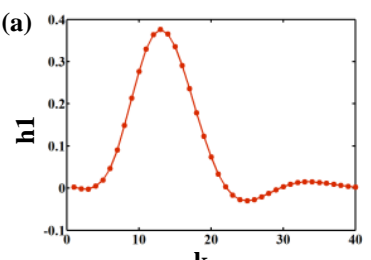

$\mathbf{k}_{1}^{20}$

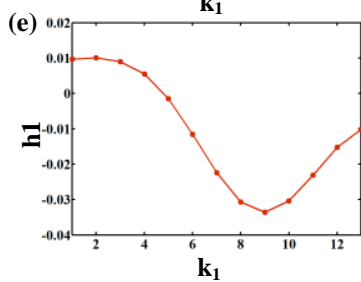

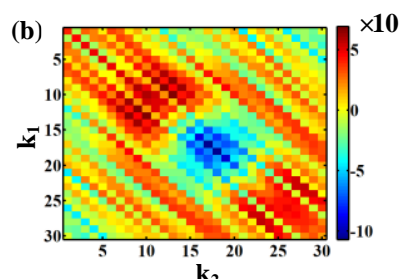

$\mathbf{k}_{2}$

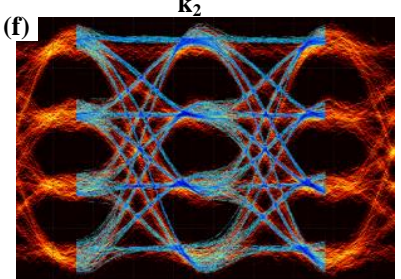

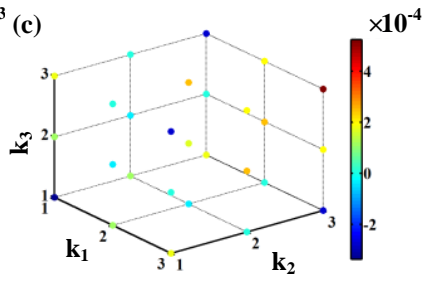

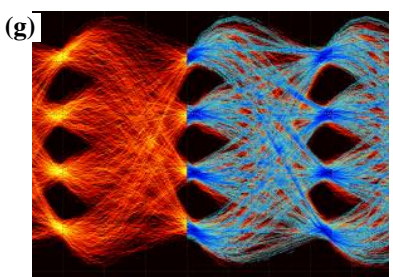

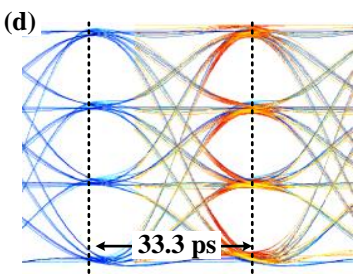

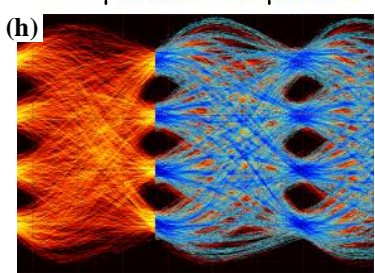

Fig. 2. (a) $1^{\text {st }}$ order, (b) $2^{\text {nd }}$ order and (c) $3^{\text {rd }}$ order kernel of the Volterra series for behavioral modeling in simulation. (d) Eye diagrams of the tested 60-Gbps PAM-4 signal (blue) in simulation and the calculated one using the estimated Volterra kernels (orange). (e) $1^{\text {st }}$ order kernel of the Volterra series for behavioral modeling in experiment. Eye diagrams of the tested PAM-4 signal (orange) in experiment and the calculated eye diagrams (blue) using the estimated Volterra kernels for (f) 20-Gbps, (g) 40-Gbps and (h) 50-Gbps PAM-4 signal. 
Fig. 1(b) shows the experimental setup for the cross-correlation-based behavioral modeling verification. The Gaussian noise signal is generated from a Keysight M8195A arbitrary waveform generator (AWG). The electrical signal is then amplified by a RF amplifier (SHF 807) to ensure that the VCSEL TOSA operates in the nonlinear region. The output electrical signal of photodetector (PD) and the electrical noise signal are both captured by a Keysight Z592A digital storage oscilloscope (DSO) with sampling rate of $160 \mathrm{GSa} / \mathrm{s}$. The cross-correlations between the received noise signal and the noise source are calculated to determine the Volterra kernels which emulate the nonlinearity of the system. In order to make the calculated eye diagrams fit well with the tested ones, the memory length is set to 13,1 and 0 for the $1^{\text {st }}, 2^{\text {nd }}$ and $3^{\text {rd }}$ order kernels, respectively. Fig. 2(c) shows the $1^{\text {st }}$ order Volterra kernels. The value of the $2^{\text {nd }}$ order Volterra kernel is -0.0092 . Fig. 2(f)-(h) show the eye diagrams for 20-, 40- and 50-Gbps PAM-4 signals. The calculated eye diagrams can reproduce the tested ones satisfactorily. Both the simulation and the experiment results indicate that the Volterra kernels estimated by cross-correlation-based behavioral modeling can emulate the nonlinearity of the transmission system using VCSEL.

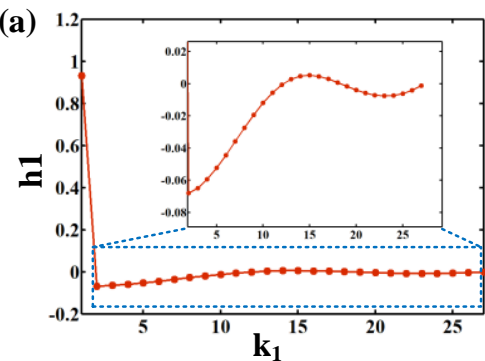

(d)

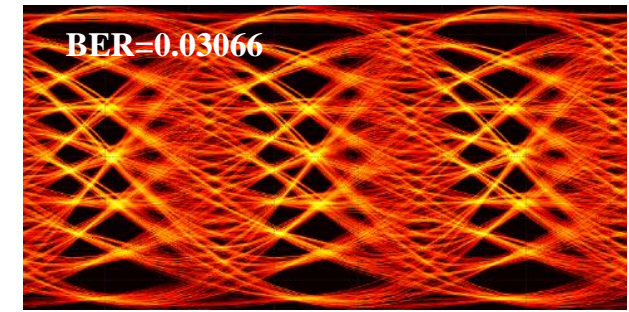

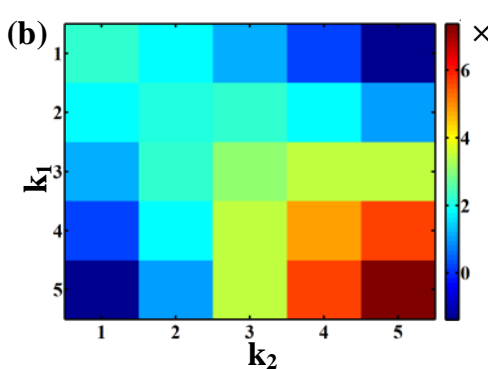

(e)

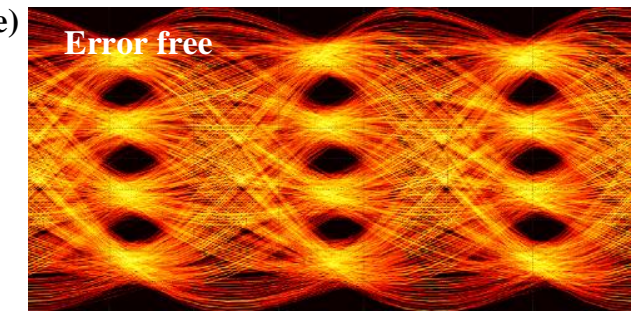

Fig. 3. (a) $1^{\text {st }}$ order kernel, (b) $2^{\text {nd }}$ order kernel and (c) $3^{\text {rd }}$ order kernel of pre-distortion Volterra series and eye diagrams of 120-Gbps PAM-4 signal (d) without pre-distortion and (e) with pre-distortion.

After verifying the effectiveness of behavioral modeling and obtaining the Volterra kernels that can emulate the transmission system, ILA is utilized to calculate the Volterra kernels of the pre-distorter for the transmission system including VCSEL and PD in VPI. The calculated pre-distortion Volterra kernels are shown in Fig. 3(a)-(c). The predistorted 120-Gbps PAM-4 signal is imported as the input of the VCSEL module in VPI. The PD output signal is shown in Fig. 3(e). Compared with the signal before pre-distortion as shown in Fig. 3(d), clear eye opening can be observed. Within the data length of the obtained signal, error free is achieved with pre-distortion applied, which indicates the validity of the proposed pre-distortion scheme for 120-Gbps VCSEL based optical interconnect.

\section{Conclusion}

In this paper, we present a novel nonlinear pre-distortion scheme based on indirect learning architecture and crosscorrelation-enabled behavioral modeling for multimode optical interconnects. 120-Gbps PAM-4 error free transmission is demonstrated using 30-GHz class VCSEL.

\section{Acknowledgement}

This work is supported in part by the National Natural Science Foundation of China under Grant 6160511.

\section{References}

[1] https://ethernetalliance.org/technology/2019-roadmap.

[2] L. Ge, et al, JLT., 37(13), pp. 3222-3228, 2019.

[3] C. Chuang et al., in $O F C, 2019$, pp. M1F.2.

[4] Q. Zhou, et al., Opt. Commun., 441, pp. 121-126, 2019.

[5] P. Berenguer et al., JLT., 34(8), pp. 1739-1745, 2016.

[6] D. Sadot, et al., in $O F C, 2018$, pp. Th3E.1.

[7] H. Faig, et al., IEEE PJ., 11(1), pp. 1-13, 2019.

[8] C. Eun and E. Powers, IEEE Trans. Signal Process., 45(1), pp. 223-227, 1997.

[9] A. Richter, S. Dris and N. André, in $O F C, 2019$, pp. Th1D.1.

[10] C. Bluemm, et al., in ECOC, 2019, pp. W3B.3.

[11] K. Szczerba and C. Kocot, in Proc SPIE, 1055204, 2018.

[12] Y. Lee and M. Schetzen, International J. Control, 2(3), pp. 237-254, 1965.

[13] J. M. Castro, et al. in $O F C$, 2016, pp. Tu2G. 2.

[14] P. Wang, J. Cartledge and W. Chan, in NUSOD 2019

[15] J. Turkiewicz et al., in ECOC, 2019, pp. Tu.1.E.3.

[16] M. Schetzen, Wiley, 1980. 